\title{
DIAGNOSTICO RADIOLOGICO DE LESIONES LUMBARES EN LA ZONA NOR-OCCIDENTAL DE HONDURAS 2014 - 2016
}

\author{
Radiological diagnosis of lumbar injuries in the north-west zones of Honduras 2014 - 2016 \\ ${ }^{\star}$ Manuel González Pieri, ${ }^{* *}$ Astrid Handal, ${ }^{* * *}$ Diana Licona, \\ ${ }^{* * * *}$ Michelle Suazo, ${ }^{* * * * *}$ Paula Villeda, ${ }^{* * * * * *}$ Yohely Rivera
}

\section{RESUMEN}

El dolor lumbar se define como un síndrome musculoesquelético cuyo principal síntoma es la presencia de dolor focalizado en el área comprendida entre la región costal inferior y la región sacra, y que en ocasiones puede comprometer la región glútea, provocando disminución funcional. Objetivo: Identificar las lesiones lumbares diagnosticadas por medio de estudio radiográfico de columna lumbar. Pacientes y Métodos: Estudio descriptivo, retrospectivo, comprendido entre 2014 y 2016 , en los pacientes que asistieron a Mediscan para la realización de estudio radiográfico de columna lumbosacra con una muestra de 687 pacientes. Resultados: De los 687 pacientes, 309(44.9\%) del género masculino, $378(55.1 \%)$ del género femenino. 211 tenían entre 51-60 años. De los pacientes del género masculino 103 (33.33\%) tenían espondiloartrosis y 63 $(20.39 \%)$ tenían canal espinal estrecho, en el género femenino $162(42.86 \%)$ tenían espondiloartrosis, $83(21.96 \%)$ discopatía lumbar. En 562 pacientes, se encontró lesión en el estudio radiológico, 264(46.9\%) a nivel de L4- L5 Conclusión: La lumbalgia se presenta más en pacientes del género femenino, con mayor frecuencia entre 51-60 años. La patología más diagnosticada fue la espondiloartrosis y el segmento más afectado fue L4-L5.

PALABRAS CLAVE: columna vertebral, dolor de la región lumbar, espondiloartritis, fractura.

\section{ABSTRACT}

Lumbar pain is defined as a musculoskeletal syndrome whose main symptom is the presence of localized pain in the area between the lower costal region and the sacral region, which may occasionally compromise the gluteal region, causing functional decline.
Objective: To identify lumbar lesions diagnosed by means of a lumbar spine radiographic study. Patient and Methods: This was a descriptive, retrospective study between 2014 and 2016 in patients who attended Mediscan for the lumbosacral spine radiographic study with a sample of 687 patients. Results: out of 687 patients, 309 (44.9\%)were males, 378 (55.1\%) were female. 211 were between $51-60$ years old. Out of male patients $103(33.33 \%)$ had spondyloarthrosis and $63(20.39 \%)$ had a narrow spinal canal, in the female gender $162(42.86 \%)$ had spondyloarthrosis, 83 (21.96\%) had lumbar disc disease. In 562 patients, a lesion was found in the radiological study, 264(46.9\%) at the L4-L5 level. Conclusion: Low back pain occurs more frequently in female patients, with a higher frequency between 51-60 years. The most diagnosed pathology was spondyloarthrosis and the segment most affected was L4-L5.

KEY WORDS: spine, low back pain, spondylorthritis, fracture.

\section{INTRODUCCION}

El dolor lumbar se define como un síndrome musculoesquelético cuyo principal síntoma es la presencia de dolor focalizado en el área comprendida entre la región costal inferior y la región sacra, y que en ocasiones puede comprometer la región glútea, provocando disminución funcional. (1)

Una de las principales causas de lumbalgia crónica ha sido la degeneración del disco intervertebral que aumenta después de los 40 años y está propiciada por una cascada de múltiples eventos celulares, inflamatorios y mecánicos complejos que condicionan inestabilidad segmentaria en la unidad funcional. ${ }^{(2,3)}$

\footnotetext{
* Doctor en medicina y cirugía, Hospital Bendaña

** Doctor en medicina y cirugía, Hospital Leonardo Martínez Valenzuela

***Doctor en medicina y cirugía, Hospital Escuela Universitario

**** Doctor en medicina y cirugía, Clínicas Por Salud

***** Doctor en medicina y cirugía, Consultorio Médico su Salud

****** Doctor en medicina y cirugía, Consultorio El Samaritano

Dirigir correspondencia a: mjpieri9@gmail.com

Recibido el: 13 de enero del 2017 aprobado 8 de mayo 2017
} 
Existen varias maneras de clasificar las lumbalgias, por ejemplo en traumáticas y congénitas (espondilolistesis, espondilólisis, fracturas, espina bífida, sacralización de L5) o agudas, subagudas y crónicas (dependiendo del tiempo de evolución de la misma). ${ }^{(3)}$

Las fracturas de la columna vertebral, ocuparon el $6 \%$ de todas las fracturas y de ellas aproximadamente el $90 \%$ ocurren en la columna dorsal y lumbar, según plantean Whang $P G$ y Vaccaro AR. (4)

Los trastornos musculo esqueléticos representaron $59 \%$ de todas las enfermedades profesionales registradas por las estadísticas europeas. (5) La lumbalgia ha sido una de las causas más frecuentes de incapacidad. En todos los momentos, entre 15 y $20 \%$ de los adultos sufren de lumbalgia. Se consideró que aproximadamente el $80 \%$ de la población mundial sufre de dolor de espalda en algún momento de su vida. ${ }^{(6,7)}$

El diagnóstico del síndrome doloroso incluye la búsqueda de los signos de alarma que traducen enfermedades sistémicas, cáncer, infecciones, fracturas, entre otras. Sin embargo en la mayoría de los pacientes la lumbalgia fue de tipo vertebral mecánica, con un curso benigno. (8) Existen diferentes métodos diagnósticos a los cuales podemos acudir para identificar signos de lesión, sin embargo debemos tener en cuenta que no son necesarios estudios de alto costo, como primera elección. En un estudio se encontró que la resonancia magnética tuvo una sensibilidad del 78 \% para la detección de espondilolistesis degenerativa de L4-L5 en comparación con el $98 \%$ para las radiografías de pie laterales. ${ }^{(9)}$

El dolor lumbar ha representado un significativo problema social y económico, incluyendo los costos médicos, disminución en la producción y cambios en la calidad de vida del que la padece. ${ }^{(10,11)}$ La radiografía simple ha sido considerada uno de los métodos diagnósticos de primera línea y con más accesibilidad económica para nuestra población. Es debido a esto y a que no se encontraron datos nacionales en bases radiográficas de las lesiones lumbares relacionadas con dolor lumbar, hemos decidido realizar el presente estudio, con el objetivo de Identificar las lesiones lumbares diagnosticadas por medio de estudio radiográfico de columna lumbar en Mediscan, durante los años 2014- 2016.

\section{PACIENTES Y METODOS:}

Estudio descriptivo, retrospectivo, comprendido entre 2014 a 2016, en los pacientes que asistieron a Mediscan para la realización de estudio radiográfico de columna lumbosacra.

El total de la población/muestra fue de 687 pacientes. Descripción de la población: La población estudiada fueron todas las personas sin distinción de género ni edad con historia de haber presentado en un momento dolor lumbar.

La máquina que se utilizó para la realización de la radiografía fue UNIVERSAL/ UNIMATIC 325 con la utilización de un buquí y casette posteriormente se pasó el casette en el Computer Radiology (CR) para la digitalización de la imagen radiográfica, y posteriormente la impresión de esta en el DRYPRO 793 y mandado digitalmente a la estación de trabajo a través del programa Osiris para la posterior interpretación por radiólogo.

Los datos que se obtuvieron de la base fueron: edad, genero, diagnostico patológico y segmento afectado. Criterios de inclusión: Pacientes a quienes se le realizo Rayos X de columna Lumbar, por causas de dolor lumbar, de todos los rangos de edades y género.

Para la tabulación de datos se utilizó el IBM SPSS stadistic program 22.

\section{RESULTADOS}

De los 687 pacientes; 309(44.9\%) eran del género masculino y $378(55.1 \%)$ del género femenino. De estos resultados como se muestra en la tabla No. 1, que la patología de mayor proporción fue la espondiloartrosis en ambos géneros con una relación 0.6:1. De los pacientes del género masculino $33.33 \%$ tenían espondiloartrosis, $20.39 \%$ tenían canal espinal estrecho y $0.32 \%$ trauma, en el género femenino $42.86 \%$ tenían espondiloartrosis, $21.96 \%$ discopatía lumbar y no se encontraron traumas. 
Tabla No. 1. Frecuencia de patología lumbar, según género.

\begin{tabular}{|c|c|c|c|c|c|}
\hline \multirow[t]{2}{*}{ Patología } & \multicolumn{2}{|c|}{ Masculino } & \multicolumn{2}{|c|}{ Femenino } & \multirow[b]{2}{*}{ TOTAL } \\
\hline & Frecuencia & Porcentaje & Frecuencia & Porcentaje & \\
\hline Espondiloartrosis & 103 & $33.33 \%$ & 162 & $42.86 \%$ & 265 (38.57\%) \\
\hline Discopatia Lumbar & 28 & $9.06 \%$ & 83 & $21.96 \%$ & $111(16.15 \%)$ \\
\hline $\begin{array}{l}\text { Canal Espinal } \\
\text { Estrecho }\end{array}$ & 63 & $20.39 \%$ & 76 & $20.11 \%$ & $139(20.23 \%)$ \\
\hline Espondilólisis & 2 & $0.65 \%$ & 2 & $0.53 \%$ & $4(0.58 \%)$ \\
\hline Espondilolistesis & 20 & $6.47 \%$ & 7 & $1.85 \%$ & $27(3.9 \%)$ \\
\hline Osteoporosis & 1 & $0.32 \%$ & 7 & $1.85 \%$ & $8(1.1 \%)$ \\
\hline Fractura & 7 & $2.27 \%$ & 0 & $0.00 \%$ & $7(1 \%)$ \\
\hline Trauma & 1 & $0.32 \%$ & 0 & $0.00 \%$ & $1(0.14 \%)$ \\
\hline Normal & 84 & $27.18 \%$ & 41 & $10.85 \%$ & $125(18 \%)$ \\
\hline Total & 309 & $100.00 \%$ & 378 & $100.00 \%$ & $687(100 \%)$ \\
\hline
\end{tabular}

Del total de pacientes con lumbalgia, 211(30.7\%) tenían entre 51-60 años. La espondiloartrosis se encontró en $59.24 \%$ de los pacientes entre $51-60$ años, en el $79.63 \%$ de los pacientes entre $61-70$ años y en $56.96 \%$ de los pacientes mayores de 70 años. La discopatía lumbar se encontró en el $27.35 \%$ de los pacientes de 41.50 años. Ver tabla No. 2.

Tabla No. 2. Frecuencia de patología lumbar y rangos de edades.

\begin{tabular}{|c|c|c|c|c|c|c|c|c|c|c|c|c|}
\hline \multirow[b]{2}{*}{ Patología } & \multicolumn{2}{|c|}{$20-30$} & \multicolumn{2}{|c|}{$31-40$} & \multicolumn{2}{|c|}{$41-50$} & \multicolumn{2}{|c|}{$51-60$} & \multicolumn{2}{|c|}{$61-70$} & \multirow{2}{*}{\multicolumn{2}{|c|}{$\begin{array}{r}>70 \\
\%\end{array}$}} \\
\hline & $\mathbf{n}$ & $\%$ & $\mathbf{n}$ & $\%$ & $\mathbf{N}$ & $\%$ & $\mathbf{n}$ & $\%$ & $\mathbf{n}$ & $\%$ & & \\
\hline Espondiloartrosis & 0 & $0.00 \%$ & 35 & $26.92 \%$ & 11 & $9.40 \%$ & 125 & $59.24 \%$ & 86 & $79.63 \%$ & 45 & $56.96 \%$ \\
\hline $\begin{array}{l}\text { Discopatía } \\
\text { Lumbar }\end{array}$ & 8 & $19.05 \%$ & 7 & $5.38 \%$ & 32 & $27.35 \%$ & 43 & $20.38 \%$ & 0 & $0.00 \%$ & 0 & $0.00 \%$ \\
\hline $\begin{array}{l}\text { Canal Espinal } \\
\text { Estrecho }\end{array}$ & 12 & $28.57 \%$ & 50 & $38.46 \%$ & 41 & $35.04 \%$ & 15 & $7.11 \%$ & 7 & $6.48 \%$ & 13 & $16.46 \%$ \\
\hline Espondilólisis & 0 & $0.00 \%$ & 2 & $1.54 \%$ & 0 & $0.00 \%$ & 1 & $0.47 \%$ & 0 & $0.00 \%$ & 0 & $0.00 \%$ \\
\hline Espondilolistesis & 0 & $0.00 \%$ & 0 & $0.00 \%$ & 0 & $0.00 \%$ & 0 & $0.00 \%$ & 6 & $5.56 \%$ & 0 & $0.00 \%$ \\
\hline Osteoporosis & 0 & $0.00 \%$ & 0 & $0.00 \%$ & 0 & $0.00 \%$ & 7 & $3.32 \%$ & 1 & $0.93 \%$ & 0 & $0.00 \%$ \\
\hline Fractura & 1 & $2.38 \%$ & & $0.00 \%$ & 1 & $0.85 \%$ & & $0.00 \%$ & & $0.00 \%$ & 12 & $15.19 \%$ \\
\hline Trauma & 1 & $2.38 \%$ & 0 & $0.00 \%$ & 0 & $0.00 \%$ & 0 & $0.00 \%$ & 0 & $0.00 \%$ & 0 & $0.00 \%$ \\
\hline Normal & 20 & $47.62 \%$ & 36 & $27.69 \%$ & 32 & $27.35 \%$ & 20 & $9.48 \%$ & 8 & $7.41 \%$ & 9 & $11.39 \%$ \\
\hline Total & 42 & & 130 & & 117 & & 211 & & 108 & & 79 & \\
\hline
\end{tabular}

Fuente: Elaboración propia del autor 
De los 562 pacientes a quienes se les encontró alteración en el estudio radiológico; 264(46.9\%) tenían la lesión a nivel de L4- L5, 185 a nivel de L5-S1 y 86 pacientes tenían la lesión a nivel de L4-L5 y S1. Ver tabla No. 3.

Tabla No. 3: Frecuencia de patología y el segmento afectado

\begin{tabular}{|c|c|c|c|c|c|c|c|c|c|c|}
\hline PATOLOGIA & $\begin{array}{l}\text { L2- } \\
13\end{array}$ & $\%$ & $\begin{array}{l}\text { L3- } \\
\text { L4 }\end{array}$ & $\%$ & $\begin{array}{l}\text { L4- } \\
\text { L5 }\end{array}$ & $\%$ & $\begin{array}{l}\text { L5- } \\
\text { S1 }\end{array}$ & $\%$ & $\begin{array}{l}\text { L4-L5- } \\
\text { S1 }\end{array}$ & $\%$ \\
\hline Espondiloartrosis & 7 & $100.00 \%$ & 6 & $30.00 \%$ & 127 & $48.11 \%$ & 84 & $45.41 \%$ & 60 & $69.77 \%$ \\
\hline $\begin{array}{l}\text { Discopatía } \\
\text { Lumbar }\end{array}$ & 0 & $0.00 \%$ & 7 & $35.00 \%$ & 61 & $23.11 \%$ & 1 & $0.54 \%$ & 13 & $15.12 \%$ \\
\hline $\begin{array}{l}\text { Canal Espinal } \\
\text { Estrecho }\end{array}$ & 0 & $0.00 \%$ & 6 & $30.00 \%$ & 49 & $18.56 \%$ & 51 & $27.57 \%$ & 4 & $4.65 \%$ \\
\hline Espondilólisis & 0 & $0.00 \%$ & 1 & $5.00 \%$ & 6 & $2.27 \%$ & 9 & $4.86 \%$ & 1 & $1.16 \%$ \\
\hline Espondilolistesis & 0 & $0.00 \%$ & 0 & $0.00 \%$ & 12 & $4.55 \%$ & 21 & $11.35 \%$ & 2 & $2.33 \%$ \\
\hline Osteoporosis & 0 & $0.00 \%$ & 0 & $0.00 \%$ & 1 & $0.38 \%$ & 1 & $0.54 \%$ & 3 & $3.49 \%$ \\
\hline Fractura & 0 & $0.00 \%$ & 0 & $0.00 \%$ & 7 & $2.65 \%$ & 18 & $9.73 \%$ & 3 & $3.49 \%$ \\
\hline Trauma & 0 & $0.00 \%$ & 0 & $0.00 \%$ & 1 & $0.38 \%$ & 0 & $0.00 \%$ & 0 & $0.00 \%$ \\
\hline Total & 7 & & 20 & & 264 & & 185 & & 86 & \\
\hline
\end{tabular}

Fuente: Elaboración Propia del autor

\section{DISCUSION}

El dolor de espalda representa un problema grave de salud, constituye un problema casi universal, entre las etiologías posibles se han citado factores físicos como el levantamiento de pesos, las cargas físicas elevadas, la vibración y el estrés postural, este último ha sido considerado como el más comúnmente implicado, puesto que incluye movimientos como inclinarse y girar, lo cuales pueden conducir a degeneración discal, dolor lumbar y ciática ${ }^{(10)}$

En este estudio se encontró que fue en el género femenino en quienes predominó el padecer de alguna patología, encontrando que $337(59.9 \%)$ de los pacientes eran mujeres y 225 (40.1\%) hombres. Similares resultados se encontraron en un estudio realizado en la ciudad de México en el hospital Ángeles Mocel durante el período de marzo de 2012 a noviembre de 2014, donde se encontró que la lumbalgia ocupa el segundo lugar de ingresos hospitalarios en el Servicio de Ortopedia y Traumatología con una frecuencia de $13.5 \%$, se estudiaron 246 pacientes mayores de 18 años con diagnóstico de lumbalgia, de los cuales 131 fueron mujeres $(53.7 \%)$ y 113 hombres $(46.3 \%)$. (11) Se encontró que $211(30.7 \%)$ de los pacientes, estaban en edades comprendidas entre 51-60 años y seguido de $130(18.9 \%)$ de pacientes que tenían edades entre 31-40 años. Inversamente a lo que refiere la literatura, donde se expresa que está considerada la principal causa de limitación de la actividad en personas menores de 45 años y la tercera en mayores de 45 años, sólo superada por la cardiopatía isquémica y otros procesos reumáticos, así como la patología músculo-esquelética más prevalente en mayores de 65 años (12)

En este estudio se obtuvo que las principales causas de dolor lumbar fueron espondiloartrosis $38.57 \%$, discopatía lumbar (16.15\%) y canal espinal estrecho $20.23 \%$, además se encontró, que según grupos de edad, la espondiloartrosis se presentó en $59.24 \%$ de los pacientes entre $51-60$ años, en el $79.63 \%$ de los pacientes entre $61-70$ años y en $56.96 \%$ de los mayores de 70 años, entre los pacientes de 31-40 años la patología más frecuente fue el canal espinal estrecho $38.46 \%$. Los resultados obtenidos, concuerdan parcialmente con el estudio realizado en México por Macías Hernández et. al, el 2014, que re vela que el diagnóstico principal por grupos de edad fue: de 18 a 30 años, hernia de disco lumbar con 25.6\%; de 31 a 40 años discopatía lumbar con $32.1 \%$; de 41 a 60 años discopatía lumbar con $30.8 \%$; de 61 a 80 años espondilolistesis con $41 \%$. (13)

El segmento lumbar más afectado en esta investigación, fue el segmento L4-L5 (38.42\%). La discopatía lumbar se encontró en 35\% a nivel de L3-L4, $23 \%$ L4-L5 y en el 15\% había afección de L4,L5 y 
S1, $48.11 \%$ de las lesiones a nivel de L4-L5 fueron espondiloartrosis y de las lesiones a nivel de L5-S1, 11.35\% fueron espondilolistesis. Estos datos son comparables con los que se presentan en la literatura, donde se describe que la hernia del núcleo pulposo ocurre en $90 \%$ de las veces en los espacios L4-L5 y L5-S1, La presentación más fre- cuente de la espondilolistesis es a nivel L5-S1. (4)

Conclusión: Lalumbalgia se presenta más en pacientes del género femenino, con mayor frecuencia entre 51-60 años. La patología más diagnosticada fue la espondiloartrosis y el segmento más afectado fue L4-L5.

\section{REFERENCIAS BIBLIOGRAFICAS}

1. Morales Morales I, Moix Queralto J, Vidal Fernandez J. Etiología, cronificación y tratamiento del dolor lumbar. Clínica y Salud.2008; 19(3):379-392.

2. Santana Rios JS, Chávez Arias DD, Coronado Zarco R, Cruz Medina E, Nava Gringas T. Tratamiento postquirúrgico de hernia discal lumbar en rehabilitación: revisión sistemática. Acta ortop. Mexicana. 2014;28(2):113-124.

3. Oliveira $\mathrm{TH}$, Oliveira VC, Melo RC, Melo RM, Freitas $\mathrm{AE}$, Ferreira $\mathrm{PH}$. Patients in treatment for chronic low back pain have higher externalised beliefs: a cross-sectional study. Rev Bras Fisioter. 2012;16(1):35-39.

4. Garro Vargas K. Lumbalgia: revisión bibliográfica. Medicina Legal de Costa Rica.[En internet] 2012 [Acceso el 22 de mayo del 2015] ;29(2):103-109. Disponible en:. http:// www.scielo.sa.cr/pdf/mlcr/v29n2/art11.pdf

5. Whang $P$, Vaccaro $R$. Thoracolumbar spine fractures and dislocations. En: Robert WBurchot, Carles M Court-broun, James D Hecherman. Rockwood and Green's Fractures in Adults. 7a ed. Philadelphia: LippicottWilliams y Wilkins. 2010;1378-1411.

6. International Labour Organization. The prevention of occupational diseases: 2 million workers killed every year. [En internet]. Geneva: International Labour Organization; 2013 [Acceso el 16 de junio del 2015]. Disponible en: http://www.ilo.org/wcmsp5/groups/ public/---ed_protect/---protrav/---safework/ documents/publication/wcms_208226.pdf

7. Lizier DT, Perez MV, Sakata RK. Ejercicios para el tratamiento de la lumbalgia inespecífica. Rev Bras Anestesiol. 2012;62(6):1-5.

8. Kuhns BD, Kouk S, Buchanan C, Lubelski D,
Alvin MD, Benzel EC. et al. Sensitivity of magnetic resonance imaging in the diagnosis of mobile and nonmobile L4-L5 degenerative spondylolisthesis. Spine J. 2015;15(9):1956-1962.

9. Martínez Suarez E. Consideraciones generales del dolor lumbar agudo. Rev. cuba. anestesiol. reanim. 2012;11(1):27-36.

10. Duque Vera IL, Zuluaga González DM, Pinilla Burgos AC. Prevalencia de lumbalgia y factores de riesgo en enfermeros y auxiliares de la ciudad de Manizales. Revista hacia la promoción de la salud. [En Internet] 2011 [Acceso el 27 de agosto del 2016];16(1):27-38. Disponible en: http://www. scielo.org.co/pdf/hpsal/v16n1/v16n1a02.pdf

11. Soto Padilla M, Espinosa Mendoza RL, Sandoval García JP, Gómez-García F. Frecuencia de lumbalgia y su tratamiento en un hospital privado de la Ciudad de México. Acta Ortop Mex. 2015; 29(1): 40-45.

12. Ocaña Jiménez Ú. Lumbalgia ocupacional y discapacidad laboral. Rev. Fisioter (Guadalupe). 2007; 6(2):17-26.

13. Macías-Hernández SI, Cruz-Medina E, Chávez-Heres T, Hernández-Herrador A, Nava-Bringas $T$, Chávez-Arias $D$, et al. Diagnóstico estructural de las lumbalgias, lumbociáticas y ciáticas en pacientes atendidos en el servicio de rehabilitación de columna del Instituto Nacional de Rehabilitación (INR). Investigación en Discapacidad.[En Internet] 2014 [Acceso el 2 de abril del 2016];3(1): 3-9. Disponible en: http://www. medigraphic.com/pdfs/invdis/ir-2014/ir141a.pdf 